

\title{
Modélisation d'un écoulement Marangoni dans une goutte en équilibre avec sa vapeur
}

\author{
Pascale Pham, Jean-Luc Achard, P.H. Masse, Jean Berthier
}

\section{To cite this version:}

Pascale Pham, Jean-Luc Achard, P.H. Masse, Jean Berthier. Modélisation d'un écoulement Marangoni dans une goutte en équilibre avec sa vapeur. La Houille Blanche - Revue internationale de l'eau, 2003, 5, pp.58-65. 10.1051/lhb/2003091 . hal-00182353

\section{HAL Id: hal-00182353 https://hal.science/hal-00182353}

Submitted on 19 Oct 2020

HAL is a multi-disciplinary open access archive for the deposit and dissemination of scientific research documents, whether they are published or not. The documents may come from teaching and research institutions in France or abroad, or from public or private research centers.
L'archive ouverte pluridisciplinaire HAL, est destinée au dépôt et à la diffusion de documents scientifiques de niveau recherche, publiés ou non, émanant des établissements d'enseignement et de recherche français ou étrangers, des laboratoires publics ou privés. 


\title{
Modélisation d'un écoulement Marangoni dans une goutte en équilibre avec sa vapeur
}

\author{
Modeling of a Marangoni flow inside a droplet \\ in equilibrium with its vapor
}

\author{
Pascale PHAM*(1) , Jean-Luc ACHARD**, Philippe MASSE***, Jean BERTHIER* \\ * Commissariat à l'Energie Atomique / LETI-DSIS, Grenoble \\ ** Laboratoire des Ecoulements Géophysiques et Industriels, Institut National Polytechnique de Grenoble \\ *** Laboratoire des Matériaux et du Génie Physique, Institut National Polytechnique de Grenoble
}

\section{RESUME}

La mise en mouvement d'un liquide sans aucun contact mécanique avec son milieu extérieur peut être un moyen de mélange et/ou brassage séduisant pour les biotechnologies où les volumes de fluide à traiter sont très petits (quelques dizaines de $\mu \mathrm{l}$ au plus). Le système présenté concerne une goutte d'eau entourée d'une enceinte fermée dont l'atmosphère est composée uniquement de sa vapeur. Une circulation dans la goutte est obtenue par effet Marangoni. L'objectif principal est de calculer le champ de vitesse dans la goutte en supposant que celle-ci est en équilibre avec sa vapeur. La goutte et sa vapeur sont considérés incompressibles, la géométrie est à symétrie $2 D$ axisymétrique, l'équation du mouvement est exprimée avec un modèle à variables non primitives $(\omega-\psi-\varphi)$. Les simulations ont permis d'étudier l'influence sur le champ de vitesse dans la goutte: (i) des phénomènes de changement de phase, (ii) de la présence de vapeur entre l'extrémité de la goutte et l'échangeur froid, (iii) du transport de chaleur par convection dans la goutte.

\section{ABSTRACT}

Inducing a convective motion inside a liquid without using any mechanical contact is a convenient and efficient way for the mixing of liquids in biotechnology, where the volumes to be treated are very small. We analyze a system constituted by a water droplet surrounded by its vapor in which a Marangoni convection has been set up. The aim of this work is to calculate the velocity field inside the droplet assuming it is at equilibrium with its vapor. Fluids are supposed incompressible and the geometry $2 D$ axisymmetric. Non primitive variables $(\omega-\psi-\varphi)$ are used in the equation governing the motion of the fluid. Numerical simulations show the influence on the droplet velocity field: (i) of the vaporization-condensation mass transfer, (ii) of the presence of vapor between the droplet and the cold source and (iii) of the convection inside the liquid.

\section{LA MICROPOMPE MARANGONI}

Les systèmes d'analyse biologique ont pour objectif la détection de biomolécules présentes dans un échantillon liquide. Les techniques actuelles consistent à mettre l'échantillon liquide en contact avec une surface sélective (surface fonctionnelle) sur laquelle les biomolécules peuvent s'accrocher par reconnaissance spécifique. Un système de lecture vient ensuite quantifier les biomolécules capturées par la surface sélective. Les normes de qualité qui s'appliquent aux industries utilisatrices de ces systèmes (industries pharmaceutiques, cosmétiques et alimentaires) sont de plus en plus rigoureuses impliquant des méthodes de détection plus rapides et plus sensibles. L'amélioration de la sensibilité de détection implique de réduire fortement la surface du détecteur (biocapteur) afin d'augmenter le rapport signal/bruit de la détection. L'augmentation de la vitesse de détection suppose, quant à elle, de diminuer le volume de l'échantillon à traiter d'une part, et de favoriser le transport des biomolécules vers de biocapteur d'autre part. Ce transport est naturellement assuré par la diffusion Brownienne. A ce mode de transport, il convient donc d'ajouter un mode de transport convectif par une mise en mouvement appropriée du liquide dans l'échantillon.

(1) PHAM Pascale, CEA, Département Systèmes pour l'Information et la Santé, Service des Systèmes pour la Biologie et la Santé, Laboratoire des Systèmes de Lecture pour la Biologie, 17 Avenue des Martyrs, 38054 Grenoble Cedex 9

email : pascale.pham@,cea.fr, tel 0438783612 


\section{I.1 Le concept}

L'idée initiale retenue était de disposer d'un moyen de créer et de contrôler un écoulement dans un volume de liquide très petit (de 10 à $100 \mu \mathrm{l}$ ) sans pièce mécanique. Des expériences de faisabilité [1] ont confirmé la possibilité de créer un écoulement organisé, dans une configuration de goutte pendante proche du microsystème que l'on veut étudier, par application d'un gradient de température. Cet écoulement, lié à l'effet Marangoni qui se manifeste sur l'interface, est utilisé ici dans un concept original désigné sous le nom de 'micropompe Marangoni' [1].

La figure ci-après donne le principe de fonctionnement de ce microsystème. L'application d'un point chaud et d'un point froid de part et d'autre de la goutte est à l'origine du gradient de température interfacial. La structure de l'écoulement permanent résultant est toroïdale. Le biocapteur étant introduit dans l'axe de la goutte, les biomolécules viennent périodiquement se rapprocher de lui afin d'être éventuellement captées dans un deuxième temps par diffusion Brownienne. Au passage notons que lorsque le temps croît indéfiniment cette seule diffusion serait suffisante pour collecter l'ensemble des biomolécules; le type de mouvement organisé qui été introduit n'est donc qu'un accélérateur de collecte ; son intérêt pratique pour les temps finis de traitement des échantillons est néanmoins essentiel.

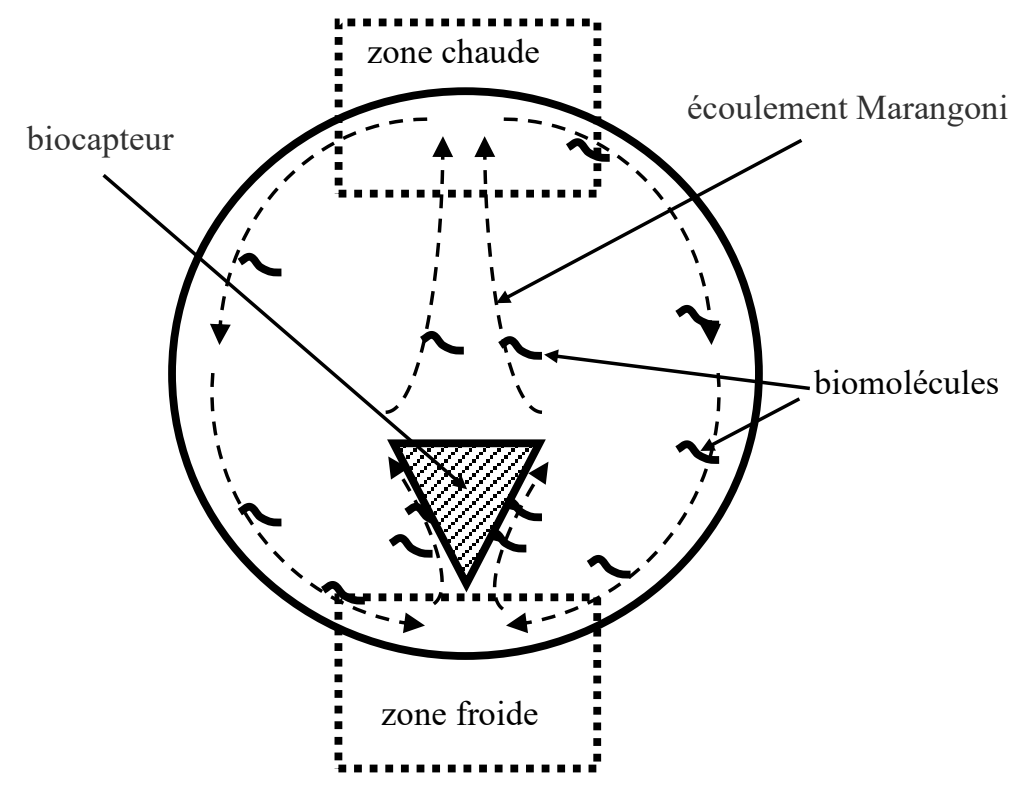

Fig. 1 : Principe de fonctionnement de la micropompe Marangoni

Tout microsystème dans lequel il existe une interface entre l'échantillon liquide à traiter et un autre fluide (liquide ou gazeux), peut jouer le rôle de micropompe Marangoni. Ainsi on peut envisager une multitude de configurations pour réaliser un tel procédé : en distinguant le système de maintien de la goutte de son système de chauffe et refroidissement, on peut citer les configurations de type goutte pendante, de type goutte posée ou encore celles où la goutte est prise entre deux plaques. Il est possible de chauffer et refroidir la goutte par contact, via le système de maintien ou directement par contact avec un élément chauffé, mais aussi à distance (utilisation d'un laser pour le chauffage et rapprochement sans contact de la partie basse de la goutte au dessus d'une surface froide par exemple). Notons que le point froid sera de préférence positionné en bas de la goutte, afin d'éviter les phénomènes de convection naturelle qui se superposeraient à l'écoulement Marangoni et risqueraient de désordonner celui-ci.

\section{I.2 La configuration de la goutte pendante}

Nous étudions ici une configuration particulière de micropompe Marangoni. Il s'agit d'une goutte d'eau d'un volume de $30 \mu \mathrm{l}$ (voir figure 2) suspendue à un tube creux (tube de suspension). Le système de refroidissement est assuré par une plaque refroidie disposée en dessous de la goutte. Le système de chauffe est assuré par contact entre la partie haute du tube de suspension et un échangeur thermique. La surface du biocapteur (surface fonctionnalisée) est la partie biseautée d'une pointe métallique introduite par le haut de la goutte. Les expériences de faisabilité [1] ont été faites sur une goutte exposée à l'air ambiant qui se vaporisait 
en quelques minutes. Le temps de capture des biomolécules étant de l'ordre de l'heure, il paraît indispensable de maîtriser les changements de phase à la surface de la goutte pour stabiliser son volume. Si la goutte est placée dans un milieu liquide non miscible (de l'huile par exemple), ces changements peuvent être évités. Si elle est plongée dans sa vapeur au sein d'une enceinte de confinement, la pression imposée dans celle-ci doit être telle que les conditions d'équilibre thermodynamique à l'interface prévalent. C'est ce deuxième cas de figure qui est abordé dans cette étude. Cependant, du fait de la présence d'une zone chaude et d'une zone froide, les conditions le long de l'interface ne sont pas isothermes et l'objectif à atteindre ici est plutôt d'obtenir un régime d'équilibre entre la masse de liquide vaporisé et la masse de vapeur condensée de part et d'autre du seul point de l'interface qui se trouve à l'équilibre thermodynamique.

La goutte, le tube de suspension, la surface froide et l'enceinte de confinement sont supposés avoir une symétrie de révolution autour de l'axe de la goutte.

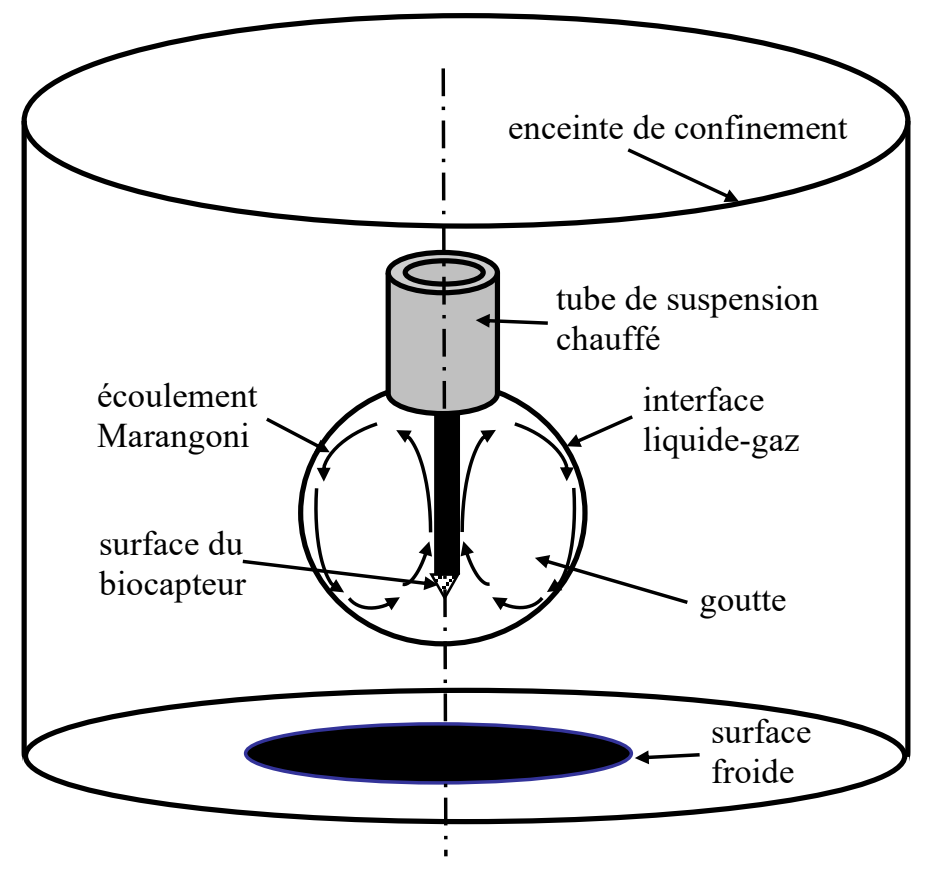

Fig. 2 : Configuration de la goutte pendante

\section{LE MODELE MICROFLUIDIQUE ( $\omega-\psi-\varphi-T)$}

Notre objectif est de mettre en place un outil de simulation numérique qui soit une aide à la réalisation d'un prototype expérimental. Le modèle doit être suffisamment flexible et englober des paramètres de commandes variés dont on pourra évaluer, lors des simulations, l'impact sur le comportement du dispositif.

Le modèle microfluidique présenté ici est issu du couplage fort entre la distribution de température $\mathrm{T}$ dans le système (équation de la chaleur) et l'écoulement dans la goutte (équations de Navier Stokes). Bien que notre objectif principal soit de connaître le champ de vitesse dans la goutte, la configuration du dispositif étudié (goutte pendante sans contact avec la source froide) nous a conduit à considérer également l'écoulement dans le gaz de l'enceinte (voir paragraphe II.1.a). Une étape de transformation des équations de la mécanique des fluides à variables primitives (vitesse $\vec{v}$ et pression p) en équations à variables non primitives (vorticité $\omega$ et fonction de courant de Stokes $\psi$ ) est avantageuse pour nos configurations où la géométrie du dispositif est à symétrie axisymétrique. Autour de ce couplage fort (modèle $\omega-\psi-T$ ), les phénomènes de vaporisationcondensation à l'interface liquide-gaz de la goutte conduisent au modèle $(\omega-\psi-\varphi-T)$ où $\varphi$ est le potentiel scalaire des vitesses obtenu par une décomposition d'Helmholtz pour la vitesse [3]. L'introduction de $\varphi$ n'est pas strictement nécessaire mais présente l'avantage de séparer les phénomènes qui agissent sur la composante normale de la vitesse à l'interface liquide-gaz (la vaporisation-condensation) des phénomènes qui agissent sur sa composante tangentielle (l'effet Marangoni). Ainsi, si dans la décomposition d'Helmholtz, on choisit de faire correspondre le potentiel vectoriel des vitesses avec la fonction de courant de Stokes $\psi$ ([1], page 182), la variable $\varphi$ sera directement associée au phénomène de changement de phase et la variable $\psi$ au phénomène Marangoni. Le modèle numérique, bien qu'alourdi par une inconnue 
supplémentaire $(\varphi)$, simplifie grandement l'interprétation des résultats en offrant la possibilité de distinguer le champ de vitesse dû au changement de phase du champ de vitesse dû à l'effet Marangoni (voir figure 6).

\section{II.1 Identification des phénomènes physiques}

La construction du modèle mathématique implique d'abord d'identifier les phénomènes physiques principaux, secondaires ou négligeables dans le système étudié qui concerne la configuration de la goutte pendante décrite par la figure 2 .

\section{a. Phénomènes physiques principaux}

Nous avons, comme phénomène physique 'dominant' ou 'principal' dans la goutte, un couplage fort entre les équations de Navier Stokes et l'équation de la thermique. Le moteur de l'écoulement est le phénomène Marangoni représenté par le gradient de température imposé le long de l'interface liquide-gaz (noté $\frac{\partial \mathrm{T}}{\partial \mathrm{s}}, \mathrm{s}$ étant l'abscisse curviligne). En retour, le champ de température et par voie de conséquence la quantité $\frac{\partial \mathrm{T}}{\partial \mathrm{s}}$, dépend du champ de vitesse dans la goutte du fait du transport de chaleur par convection. Un second phénomène principal provient de la présence de vapeur entre la surface froide et le bas de la goutte : cette vapeur joue un rôle de résistance thermique caractérisée par une conductibilité thermique $\lambda$ faible devant celle de l'eau. La température effective du bas de la goutte $\mathrm{T}_{\text {bas }}$, dont dépend directement la quantité $\frac{\partial \mathrm{T}}{\partial \mathrm{s}}$, est très différente de la température imposée sur la surface froide $T_{\text {froid. }}$.

\section{b. Phénomènes physiques secondaires}

Les phénomènes physiques secondaires sont les phénomènes induits dans le système piloté par les phénomènes principaux. Ils sont donc soit la conséquence du mouvement dans la goutte (cas de la convection thermique dans la vapeur), soit la conséquence directe de l'application d'un écart de température sur le système (cas de la vaporisation-condensation à la surface de la goutte). Leur influence sur le champ de vitesse dans la goutte est difficilement quantifiable par des estimations globales, le modèle se révèle ici très précieux.
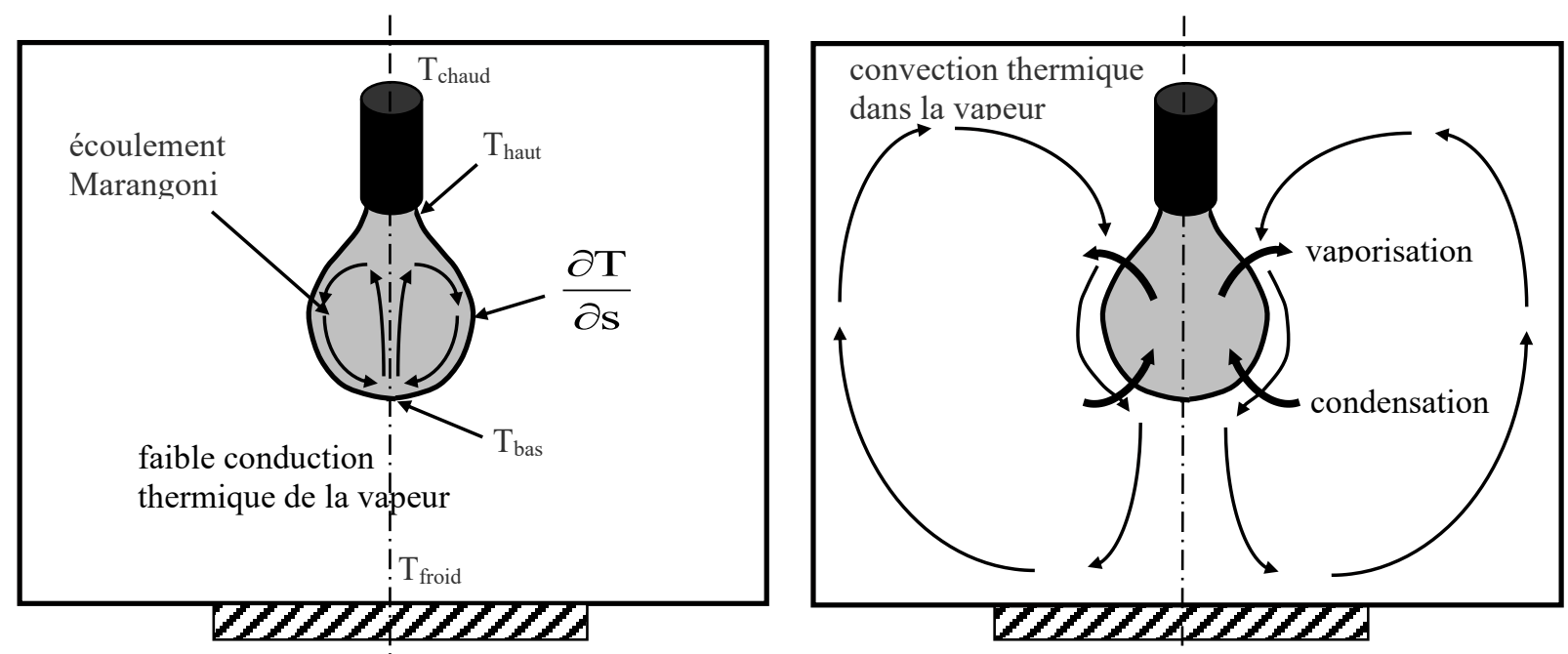

Fig. 3 : Phénomènes physiques principaux (gauche) et secondaires (droite)

\section{c. Phénomènes physiques négligeables}

Concernant les phénomènes négligeables nous pouvons citer :

- Le phénomène Marangoni chimique qui serait provoqué par la présence d'un tensioactif dans la goutte [1] et qui tend à s'opposer à la cause du mouvement qui lui a donné naissance, à savoir le phénomène Marangoni thermique. Nous avons exclu dans cette étude la présence de tensioactif dans la goutte constituée d'eau pure. 
- Le phénomène de convection naturelle qui peut être à l'origine d'instabilités dans l'écoulement: en positionnant la zone froide sur la partie basse de la goutte, ces instabilités peuvent être inhibées. L'analyse dimensionnelle, conduite dans [1], montre que les forces de flottabilité restent négligeables dans notre cas.

- La forme et la taille de la goutte dépendent des conditions de suspension de la goutte, à savoir le rayon du tube de suspension, la hauteur de liquide dans ce tube, les conditions de pression extérieure qui, dans notre cas, sont les conditions de pression imposées dans l'enceinte de confinement. L'analyse dimensionnelle effectuée dans [1], a montré que la forme de la goutte ne dépend pas des forces visqueuses qui résultent du mouvement. La forme de la goutte est donc la même que celle issue de conditions hydrostatiques et elle a été estimée ici à partir des outils de calcul développés dans [2].

\section{II.2 Equations et conditions aux limites du modèle microFluidique $(\omega-\psi-\varphi-T)$}

Les équations que nous utiliserons ici sont exprimées dans le système d'Unités International.

\section{II.2.1 Modèle à variables primitives $\left(v_{r}, v_{z}, p, T\right)$}

\section{a. Equations}

Nous appelons 'variables primitives' les variables vitesse $\vec{v}$, pression $p$ et température T. L'analyse dimensionnelle effectuée dans [1] a permis d'établir les équations de Navier Stokes dans lesquelles les termes relatifs à la variation de la viscosité avec la température d'une part et le terme relatif à la gravité d'autre part sont négligés. Ainsi l'équation issue de la conservation de la quantité de mouvement associée à l'équation de conservation de la masse donnent le système suivant :

$$
\left\{\begin{array}{l}
\rho_{0}(\overrightarrow{\mathrm{v}} \cdot \vec{\nabla}) \overrightarrow{\mathrm{v}}+\vec{\nabla} \mathrm{p}-\mu_{0} \Delta \overrightarrow{\mathrm{v}}=0 \\
\vec{\nabla} \cdot \overrightarrow{\mathrm{v}}=0
\end{array}\right.
$$

où $\mathrm{p}$ est la pression dans le fluide et $\overrightarrow{\mathrm{v}}$ sa vitesse. L'indice ${ }_{0}$ qui apparait devant la densité $\rho$ et la viscosité dynamique $\mu$ du fluide indique que ces propriétés doivent être estimées pour une température de référence $T_{0}$ qui est la température médiane entre $T_{\text {haut }}$ et $T_{\text {bas }}$ appliquées aux deux extrémités de l'interface liquide-gaz de la goutte (voir figure 3 gauche). Ce système d'équations est valable à la fois dans le liquide (indice i) et dans le gaz (indice e).

La prise en compte de l'effet Marangoni implique un couplage fort entre ces équations et l'équation de la thermique justifiée par l'analyse dimensionnelle de [1] :

$$
\rho_{0} \mathrm{C}_{\mathrm{p}}(\overrightarrow{\mathrm{v}} \cdot \vec{\nabla} \mathrm{T})+\vec{\nabla} \cdot(-\lambda \vec{\nabla} \mathrm{T})=0
$$

où $\mathrm{C}_{\mathrm{p}}$ est la capacité calorifique du fluide supposée indépendante de la température, $\lambda$ sa conductibilité thermique.

\section{b. Conditions aux limites}

Les conditions aux limites associées aux équations (1) et (2) se distinguent selon la nature des frontières :

- Sur l'interface liquide-gaz: on utilise sur cette frontière le repère intrinsèque $(\overrightarrow{\mathrm{n}}, \overrightarrow{\mathrm{s}})$ constitué du vecteur normal $\vec{n}$ et de son vecteur tangent $\vec{s}$ [1]. La contrainte Marangoni provient de la variation de tension superficielle le long de l'interface (notée $\frac{\partial \sigma}{\partial s}$ ) ; elle est équilibrée par les composantes tangentielles des contraintes visqueuses qui apparaissent dans chaque fluide. Les deux fluides sont ainsi mis en mouvement au voisinage de cette interface. Le bilan tangentiel de quantité de mouvement qui traduit cet équilibre donne :

$$
\overrightarrow{\mathrm{n}} \cdot \tau_{\mathrm{i}} \cdot \overrightarrow{\mathrm{s}}-\overrightarrow{\mathrm{n}} \cdot \tau_{\mathrm{e}} \cdot \overrightarrow{\mathrm{s}}=\frac{\partial \sigma}{\partial \mathrm{s}}=-\gamma \frac{\partial \mathrm{T}}{\partial \mathrm{s}}
$$

où $\stackrel{\bar{\tau}}{\tau}$ est le tenseur des contraintes visqueuses dans la goutte (indice i) et dans la vapeur (indice e). La tension superficielle $\sigma$ apparaît dans la zone qui nous intéresse comme dépendant linéairement de $\mathrm{T}$ fonction et l'on définit la grandeur $\gamma$ par :

$$
\gamma=-\frac{\partial \sigma(\mathrm{T})}{\partial \mathrm{T}}
$$

L'adhérence des fluides entre eux se traduit par la continuité de leur vitesse tangentielle :

$$
\overrightarrow{\mathrm{v}}_{\mathrm{e}} \cdot \overrightarrow{\mathrm{s}}=\overrightarrow{\mathrm{v}}_{\mathrm{i}} \cdot \overrightarrow{\mathrm{s}}
$$


Si l'on désigne par $\dot{\mathrm{m}}$ le taux de masse de liquide ou de vapeur échangé à l'interface liquide-gaz par vaporisation-condensation, on peut dire, qu'à tout instant, le bilan de masse se traduit par :

$$
\dot{\mathrm{m}}=\rho_{0 \mathrm{i}} \overrightarrow{\mathrm{v}}_{\mathrm{i}} \cdot \overrightarrow{\mathrm{n}}_{\mathrm{i}}=-\rho_{0 \mathrm{e}} \overrightarrow{\mathrm{v}}_{\mathrm{e}} \cdot \overrightarrow{\mathrm{n}}_{\mathrm{e}}
$$

La relation (5) suppose que la vitesse de déplacement de l'interface liquide-gaz est nulle ce qui est le cas si le profil de la goutte est stable au cours du temps. En l'absence de changement de phase, l'équation (5) se transforme, pour les deux fluides, en condition d'imperméabilité :

$$
\overrightarrow{\mathrm{v}}_{\mathrm{i}} \cdot \overrightarrow{\mathrm{n}}_{\mathrm{i}}=\overrightarrow{\mathrm{v}}_{\mathrm{e}} \cdot \overrightarrow{\mathrm{n}}_{\mathrm{e}}=0
$$

Si les études fines montrent, qu'en présence d'un changement de phase, un liquide n'a pas la même température que sa vapeur au niveau de l'interface qui les sépare, on admettra en première approximation la continuité des températures :

$$
\mathrm{T}_{\mathrm{i}}=\mathrm{T}_{\mathrm{e}}=\mathrm{T}_{\mathrm{s}}
$$

où l'on note $\mathrm{T}_{\mathrm{s}}$ la température de l'interface liquide-gaz. Dit autrement, nous négligeons le saut de température interfacial dû au déséquilibre thermique. En revanche, nous n'adoptons pas l'hypothèse dite d'équilibre chimique qui résulte de l'égalité de l'enthalpie libre de part et d'autre de l'interface. Cet équilibre se traduit sous sa forme classique par:

$$
\mathrm{p}_{\mathrm{e}}=\mathrm{p}_{\mathrm{sat}}\left(\mathrm{T}_{\mathrm{s}}\right)
$$

où $\mathrm{p}_{\mathrm{e}}$ est la pression dans la vapeur et $\mathrm{p}_{\mathrm{sat}}$ la pression de vapeur saturante donnée par la courbe de vaporisation (loi de Clausius-Clapeyron). L'hypothèse d'équilibre thermodynamique suppose à la fois (8) et (9). Cette hypothèse est relaxée et l'on utilise, à la place de (9), la célèbre loi de Hertz-Knudsen-Schrage qui permet d'exprimer le taux de masse $\dot{m}$ [1] :

$$
\dot{\mathrm{m}}=\frac{2}{2-\alpha_{\mathrm{c}}} \frac{1}{\left(2 \pi \Re \mathrm{T}_{\mathrm{s}}\right)^{\frac{1}{2}}}\left[\alpha_{\mathrm{c}} \mathrm{p}_{\mathrm{e}}-\alpha_{\mathrm{v}} \mathrm{p}_{\mathrm{sat}}\left(\mathrm{T}_{\mathrm{s}}\right)\right]
$$

où $\mathfrak{R}$ est la constante des gaz parfaits divisée par la masse molaire de la vapeur, $\alpha_{c}$ et $\alpha_{v}$ des coefficients déterminés empiriquement et appelés coefficients d'accommodations.

Concernant la loi de conservation de l'énergie à l'interface liquide-gaz, nous supposons que le saut de flux de chaleur y est dû uniquement au changement de phase, caractérisé par une chaleur latente L [1] :

$$
-\lambda_{\mathrm{i}} \vec{\nabla} \mathrm{T}_{\mathrm{i}} \cdot \overrightarrow{\mathrm{n}}_{\mathrm{i}}-\lambda_{\mathrm{e}} \vec{\nabla} \mathrm{T}_{\mathrm{e}} \cdot \overrightarrow{\mathrm{n}}_{\mathrm{e}}=\dot{\mathrm{m}} \mathrm{L}
$$

- Sur les parois solides : on exprime, sur ces parois, la condition d'adhérence du fluide et d'imperméabilité, à savoir:

$$
\overrightarrow{\mathrm{v}}_{\mathrm{k}} \cdot \overrightarrow{\mathrm{n}}=\overrightarrow{\mathrm{v}}_{\mathrm{k}} \cdot \overrightarrow{\mathrm{s}}=0, \mathrm{k}=\mathrm{i}, \mathrm{e}
$$

Côté thermique, les conditions aux limites sont soit de type Dirichlet sur les parois en contact avec les échangeurs de chaleur (température imposée $T_{\text {chaud }}$ ou $T_{\text {froid }}$ ), soit de type Neumann homogène pour les autres parois solides considérées adiabatiques (parois de l'enceinte de confinement...) :

$$
-\lambda_{\mathrm{k}} \vec{\nabla} \mathrm{T}_{\mathrm{k}} \cdot \overrightarrow{\mathrm{n}}=0, \mathrm{k}=\mathrm{i}, \mathrm{e}
$$

\section{II.2.2 Modèle à variables non primitives $(\omega-\psi-\varphi-T)$}

\section{a. Equations $(\omega-\psi-\varphi)$}

Dans le cas d'une symétrie axisymétrique, il est intéressant de remplacer les variables primitives citées cidessus par les variables non primitives $\omega$ (vorticité) et $\psi$ (fonction de courant) : l'équation de conservation de la masse est automatiquement vérifiée et le nombre d'inconnues du système à résoudre est diminué. Nous utilisons ici la fonction de courant $\psi$ définie selon Happel et Brenner [4] :

$$
\mathrm{v}_{\mathrm{r}}=\frac{1}{\mathrm{r}} \frac{\partial \psi}{\partial \mathrm{z}} ; \mathrm{v}_{\mathrm{z}}=-\frac{1}{\mathrm{r}} \frac{\partial \psi}{\partial \mathrm{r}}
$$

Pour la vorticité $\omega$ :

$\vec{\omega}=\vec{\nabla} \times \vec{v}$

Pour représenter le phénomène de vaporisation-condensation à l'interface liquide-gaz, nous introduisons une décomposition d'Helmholtz de la vitesse [3] :

$$
\overrightarrow{\mathrm{v}}=\vec{\nabla} \varphi+\vec{\nabla} \times \overrightarrow{\mathrm{A}}
$$


où $\overrightarrow{\mathrm{A}}$ est appelé potentiel vectoriel des vitesses et $\varphi$ potentiel scalaire des vitesses. La décomposition d'Helmholtz n'étant pas unique, nous choisissons de faire correspondre le potentiel vecteur $\vec{A}$ avec la fonction de courant $\psi$ définie par (14) :

$$
\overrightarrow{\mathrm{A}}=-\frac{\psi}{\mathrm{r}} \overrightarrow{\mathrm{u}}_{\theta}
$$

L'introduction des variables non primitives et de leur définition dans le système d'équations (1) aboutit au système suivant $[1]$ :

$$
\left\{\begin{array}{c}
\Delta \varphi=0 \\
(\overrightarrow{\mathrm{v}} . \vec{\nabla}) \omega-v_{0}\left[\Delta \omega-\frac{\omega}{\mathrm{r}^{2}}\right]=0 \\
\frac{1}{\mathrm{r}} \mathrm{E}^{2} \psi=\omega
\end{array}\right.
$$

où $v$ est la viscosité cinématique et la vitesse est telle que :

$$
\overrightarrow{\mathrm{v}}=\left(\frac{\partial \varphi}{\partial \mathrm{r}}+\frac{1}{\mathrm{r}} \frac{\partial \psi}{\partial \mathrm{z}}\right) \overrightarrow{\mathrm{u}}_{\mathrm{u}}+\left(\frac{\partial \varphi}{\partial \mathrm{z}}-\frac{1}{\mathrm{r}} \frac{\partial \psi}{\partial \mathrm{r}}\right) \overrightarrow{\mathrm{u}}_{\mathrm{z}}
$$

Le modèle $(\omega-\psi-\varphi)$ définit par $(18)$ reste couplé à l'équation de thermique (2) pour donner le modèle $(\omega-\psi-$ $\varphi-T)$.

\section{b. Conditions aux limites associées}

- Sur l'interface liquide-gaz, la traduction du bilan tangentiel de quantité de mouvement (3) avec la décomposition d'Helmholtz de la vitesse devient [1] :

$$
\omega_{\mathrm{i}}=\frac{1}{\mu_{0 \mathrm{i}}}\left[-2 \frac{\partial \dot{\mathrm{m}}}{\partial \mathrm{s}}\left(\frac{\mu_{0 \mathrm{e}}}{\rho_{0 \mathrm{e}}}+\frac{\mu_{0 \mathrm{i}}}{\rho_{0 \mathrm{i}}}\right)+\mu_{0 \mathrm{e}} \omega_{\mathrm{e}}-\gamma \frac{\partial \mathrm{T}}{\partial \mathrm{s}}\right]
$$

La condition d'entrainement de la vapeur par le liquide (5) donne une condition sur $\psi$ côté vapeur :

$$
\frac{\partial \psi_{\mathrm{e}}}{\partial \mathrm{n}_{\mathrm{e}}}=\mathrm{r}\left(\frac{\partial \varphi_{\mathrm{i}}}{\partial \mathrm{s}}-\frac{\partial \varphi_{\mathrm{e}}}{\partial \mathrm{s}}\right)+\frac{\partial \psi_{\mathrm{i}}}{\partial \mathrm{n}_{\mathrm{i}}}
$$

Alors que le phénomène de vaporisation-condensation (6) se traduit en une condition sur la variable $\varphi$ :

$$
\rho_{0 \mathrm{i}} \vec{\nabla} \varphi_{\mathrm{i}} \cdot \overrightarrow{\mathrm{n}}_{\mathrm{i}}=-\rho_{0 \mathrm{e}} \vec{\nabla} \varphi_{\mathrm{e}} \cdot \overrightarrow{\mathrm{n}}_{\mathrm{e}}=\dot{\mathrm{m}}
$$

- Sur les parois solides : avec la condition d'imperméabilité (7) couplée à la condition (22) où le taux de masse échangé est nul, nous avons :

$$
\frac{\partial \psi_{\mathrm{i}}}{\partial \mathrm{s}}=\frac{\partial \psi_{\mathrm{e}}}{\partial \mathrm{s}}=0
$$

alors que la condition d'adhérence devient :

$$
\left\{\begin{array}{l}
\frac{\partial \psi_{\mathrm{i}}}{\partial \mathrm{n}_{\mathrm{i}}}=-\mathrm{r} \frac{\partial \varphi_{\mathrm{i}}}{\partial \mathrm{s}} \\
\frac{\partial \psi_{\mathrm{e}}}{\partial \mathrm{n}_{\mathrm{e}}}=-\mathrm{r} \frac{\partial \varphi_{\mathrm{e}}}{\partial \mathrm{s}}
\end{array}\right.
$$

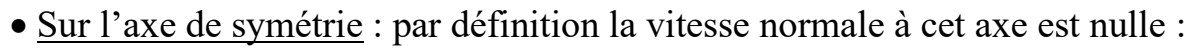

$\vec{\nabla} \varphi_{\mathrm{i}} \cdot \overrightarrow{\mathrm{n}}_{\mathrm{i}}=\vec{\nabla} \varphi_{\mathrm{e}} \cdot \overrightarrow{\mathrm{n}}_{\mathrm{e}}=0$

De plus, la définition (14) de la fonction de courant et l'absence de contrainte tangentielle le long de cet axe imposent :

$$
\psi=\omega=0
$$

\section{ETUDE NUMERIQUE DE LA CONFIGURATION DE LA GOUTTE PENDANTE}

Le modèle $(\omega-\psi-\varphi-T)$ décrit dans le paragraphe II.2.2 a été résolu par la Méthode des Eléments Finis (logiciel Flux-Expert [5]) sur la configuration de la goutte pendante. Nous choisissons un cas de référence à partir duquel nous étudierons l'influence du changement de phase et de la convection dans le liquide sur le 
champ de vitesse dans la goutte. Les paramètres de modélisation sont les dimensions géométriques, les valeurs des paramètres physiques qui apparaissent dans les équations à résoudre (cf. II) et l'écart de température $\left(\Delta \mathrm{T}_{\max }=\mathrm{T}_{\text {chaud }}-\mathrm{T}_{\text {froid }}\right)$ appliqué au système.

\section{III.1 Paramètres de modélisation}

La figure 4 donne la description de la géométrie du cas de référence dans une symétrie axisymétrique. La forme de la goutte est issue de [2] : son rayon, pris sur sa partie la plus large, est de $1.8 \mathrm{~mm}$, sa longueur $\mathrm{H}_{\mathrm{i}}$ est de $3.5 \mathrm{~mm}$ et son volume est de $29 \mu \mathrm{l}$. Le rayon $\mathrm{R}$ du tube de suspension est de $1 \mathrm{~mm}$. Ces dimensions respectent approximativement le critère de stabilité de la goutte pendante pour lequel la longueur de la goutte est environ deux à trois fois le rayon $\mathrm{R}$ du tube de suspension [2]. Afin d'être dans un cas de figure compatible avec l'hypothèse où la variation de la viscosité de l'eau avec la température est négligeable, nous imposons une distance $\mathrm{H}_{\mathrm{e}}$ entre la goutte et l'échangeur froid de $2 \mathrm{~mm}$. Le tube de suspension est en acier inoxydable et la pointe chauffante est en cuivre.
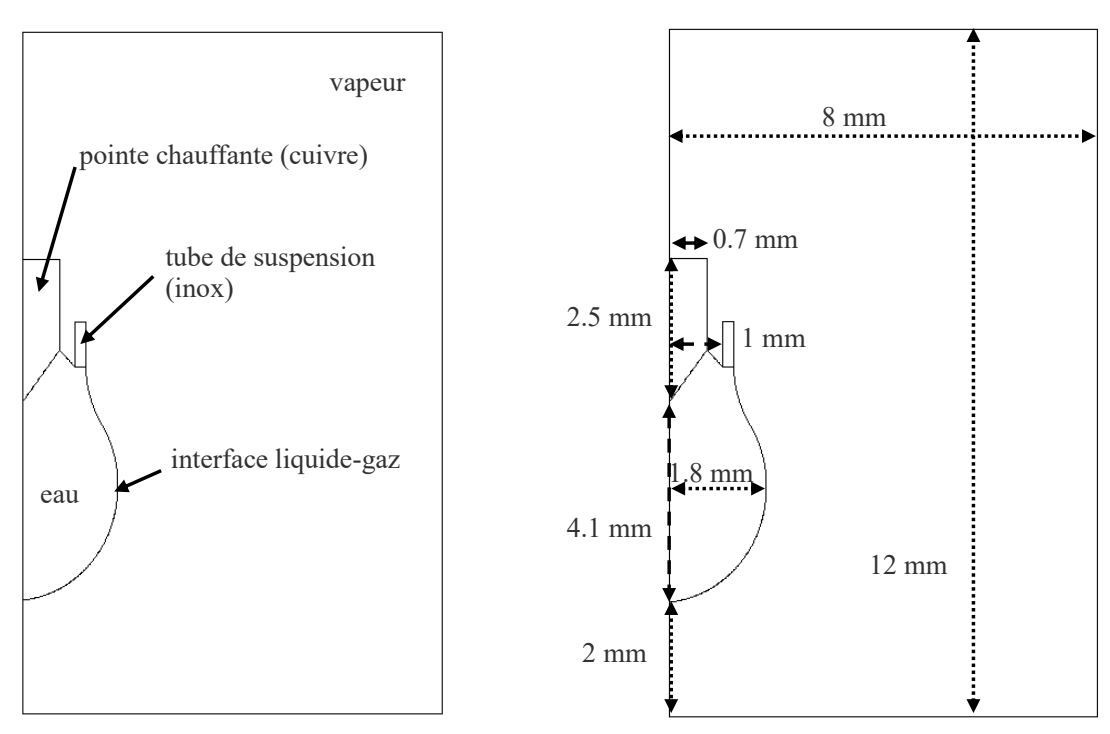

Fig. 4 : Géométrie de modélisation et dimensions du cas de référence

Les propriétés des matériaux sont la conductibilité thermique, la capacité calorifique, la masse volumique et la viscosité dynamique des différents constituants du système. D'après [1], nous avons :

\begin{tabular}{|c|c|c|c|c|}
\hline & $\begin{array}{c}\text { conductibilité thermique } \\
\lambda(\mathrm{W} / \mathrm{m} / \mathrm{K})\end{array}$ & $\begin{array}{c}\text { capacité calorifique } \\
\mathrm{C}_{\mathrm{p}}(\mathrm{J} / \mathrm{K} / \mathrm{Kg})\end{array}$ & $\begin{array}{c}\text { viscosité dynamique } \\
\mu(\mathrm{Kg} / \mathrm{m} / \mathrm{s})\end{array}$ & $\begin{array}{c}\text { masse volumique } \\
\rho\left(\mathrm{Kg} / \mathrm{m}^{3}\right)\end{array}$ \\
\hline eau & $0.57\left(20^{\circ} \mathrm{C}\right)$ & $4187\left(20^{\circ} \mathrm{C}\right)$ & $10^{-3}\left(20^{\circ} \mathrm{C}\right)$ & $1000\left(20^{\circ} \mathrm{C}\right)$ \\
\hline vapeur & $0.024\left(20^{\circ} \mathrm{C}\right)$ & $1856\left(20^{\circ} \mathrm{C}\right)$ & $2.1410^{-5}\left(25^{\circ} \mathrm{C}\right)$ & $0.068\left(46^{\circ} \mathrm{C}\right)$ \\
\hline $\begin{array}{c}\text { inox (tube de } \\
\text { suspension) }\end{array}$ & $50\left(20^{\circ} \mathrm{C}\right)$ & - & - & - \\
\hline $\begin{array}{c}\text { cuivre (pointe } \\
\text { chauffante) }\end{array}$ & $390\left(20^{\circ} \mathrm{C}\right)$ & - & - & - \\
\hline
\end{tabular}

Tableau 1 : Propriétés thermo-fluidiques des différents constituants du cas de référence

Notons que nous n'avons pas besoin de connaître la masse volumique et la capacité calorifique des milieux solides puisque ces deux paramètres interviennent uniquement dans le terme de convection de l'équation thermique (2); terme qui s'annule du fait d'une vitesse nulle dans ces parties solides.

Concernant la tension superficielle $\sigma$ de l'eau et sa variation avec la température $\left(\gamma=-\frac{\partial \sigma}{\partial T}\right)$, notons que la littérature n'est pas toujours en accord sur la valeur de $\gamma$. Ce paramètre étant central dans notre modèle, nous l'avons estimé dans [1] directement à partir des valeurs de tension superficielle mesurées à différentes températures : entre 0 et $100^{\circ} \mathrm{C}$, la tension superficielle de l'eau varie linéairement avec la température et donne une valeur de $\gamma$ d'environ $0.15 \mathrm{mN} / \mathrm{m} / \mathrm{K}$. 
Les paramètres de vaporisation-condensation qui apparaissent dans la loi de Hertz-Knudsen-Schrage (10) concernent les coefficients d'accommodation de vaporisation et de condensation, $\alpha_{v}$ et $\alpha_{c}\left(\alpha_{v}=\alpha_{c}=0.04\right.$ d'après [1]), la pression imposée dans l'enceinte $p_{e}$, et la courbe de saturation $p_{\text {sat }}(T)$ de l'eau qui peut être représentée par la fonction mathématique $\mathrm{p}_{\text {sat }}=(\mathrm{T} / 100)^{4}$ où $\mathrm{p}_{\text {sat }}$ est en atm et $\mathrm{T}$ en degrés Celsius. Dans (10), $\mathfrak{R}$ est la constante des gaz parfaits divisée par la masse molaire de la vapeur qui, pour l'eau, vaut 47.06 $\mathrm{J} / \mathrm{K} / \mathrm{Kg}$ [1]. Le taux de masse vaporisé ou condensé $\dot{\mathrm{m}}$ dépend de la température de l'interface liquide-gaz $\mathrm{T}_{\mathrm{s}}$ déterminée en tout point de l'interface liquide-gaz. Ce taux de masse intervient dans (11) où $\mathrm{L}$ est la chaleur latente de vaporisation de l'eau égale à $2.2510^{6} \mathrm{~J} / \mathrm{Kg}$. Les températures des échangeurs de chaleur sont choisies telles que $\mathrm{T}_{\text {chaud }}=30^{\circ} \mathrm{C}$ et $\mathrm{T}_{\text {froid }}=10^{\circ} \mathrm{C}$.

\section{III.2 Résultats de simulation du cas de référence}

Les différents paramètres de simulation du modèle microfluidique $(\omega, \psi, \varphi, T)$ étant définis, nous présentons le champ de vitesse et de température dans la goutte et sa vapeur pour le cas de référence où les phénomènes de vaporisation-condensation sont négligés :
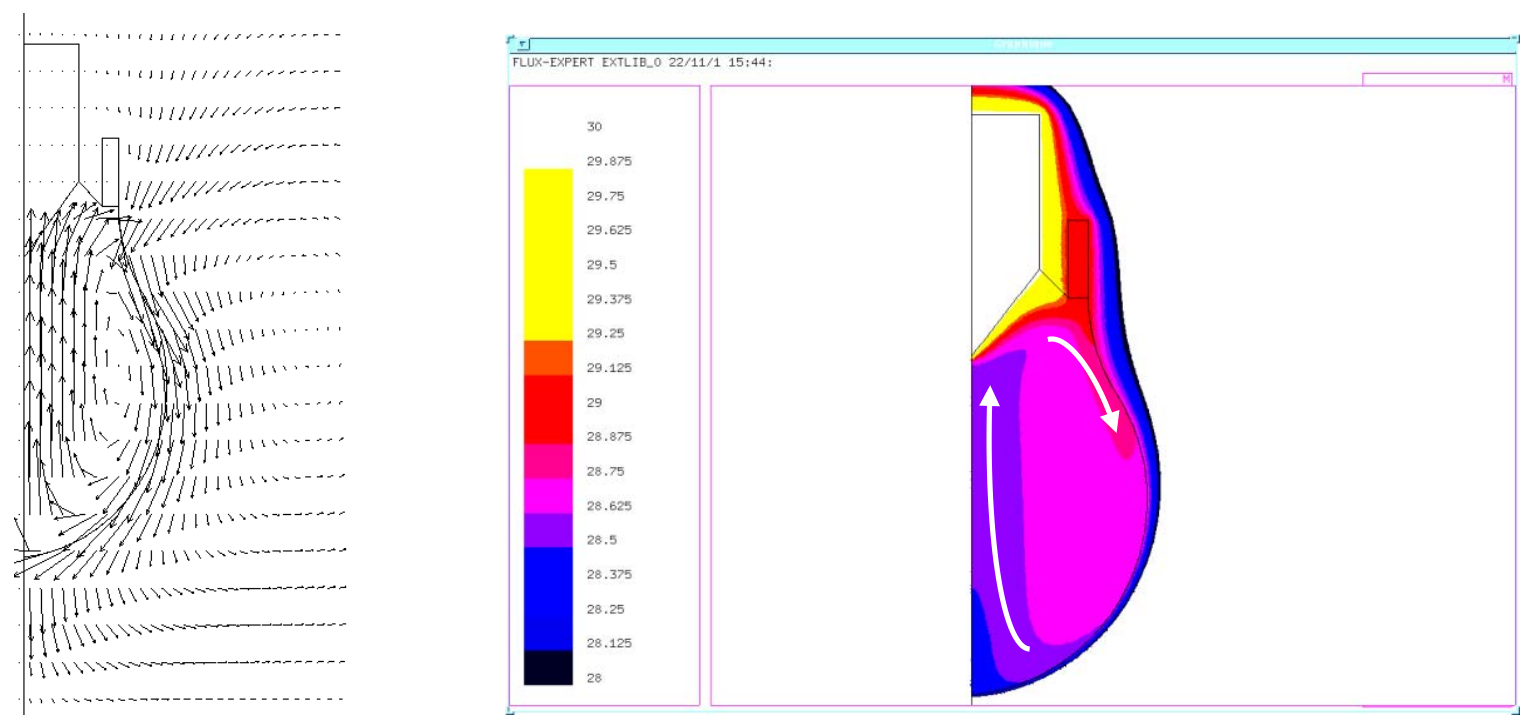

Fig. 5 : Champs de vitesse (gauche, Vmax $=8.6 \mathrm{~mm} / \mathrm{s}$ ) et de température (droite) pour le cas de référence

On voit que l'écoulement est ascendant le long de l'axe de symétrie du système et descendant le long de l'interface liquide-gaz (figure 5 gauche). Ces deux zones constituent les régions où la vitesse est la plus forte $\left(\mathrm{V}_{\max }=8.6 \mathrm{~mm} / \mathrm{s}\right)$. Le sens de l'écoulement est imposé de par le positionnement des deux échangeurs de chaleur, à savoir l'échangeur chaud situé sur la partie haute du système et l'échangeur froid sur sa partie basse. La remontée de liquide le long de l'axe de symétrie a lieu sur une veine assez large, ce qui est en accord avec les observations expérimentales [1]. L'entraînement de la vapeur par contact avec la goutte a bien lieu et on perçoit l'écoulement qui en résulte. Sur la figure 5 droite, les zones sombres correspondent aux zones froides alors que les zones claires correspondent aux zones chaudes. Cette carte d'isothermes montre bien l'effet de convection important dans la goutte qui se traduit par une remontée de liquide froid le long de l'axe de symétrie et un entraînement du liquide chaud le long de l'interface liquide-gaz. Notons enfin que si l'écart de température imposé au système est de $20^{\circ} \mathrm{C}\left(\mathrm{T}_{\text {chaud }}-\mathrm{T}_{\text {froid }}\right)$, l'écart de température qui est appliqué à l'interface liquide-gaz n'est plus que de $0.7^{\circ} \mathrm{C}\left(\mathrm{T}_{\text {haut }}-\mathrm{T}_{\text {bas }}\right)$ confirmant ainsi l'effet important de résistance thermique de la vapeur cité plus haut.

\section{a. Influence de la convection dans la goutte}

Pour évaluer l'influence du transport de chaleur par convection dans la goutte du fait de l'effet Marangoni, il suffit de reprendre la simulation précédente et d'annuler le terme de convection dans l'équation de la thermique (2) pour l'eau. Les résultats obtenus aboutissent à une vitesse maximale de $136 \mathrm{~mm} / \mathrm{s}$ dans la goutte et un écart de température à l'interface liquide-gaz de $3.1^{\circ} \mathrm{C}$. Ces résultats montrent bien que la convection dans la goutte affaiblit notablement le moteur Marangoni en atténuant fortement le gradient de température le long de l'interface liquide-gaz. 


\section{b. Influence de la vaporisation-condensation autour de la goutte}

Rappelons que la prise en compte du changement de phase donne une valeur non nulle de la variable $\varphi$ dans notre modèle. La figure 6(a) montre le champ de vitesse obtenu dans la goutte et dans la vapeur (a) et la figure 6(b) la composante de la vitesse qui ne concerne que ce phénomène de changement de phase à savoir $\vec{\nabla} \varphi:$

(a)

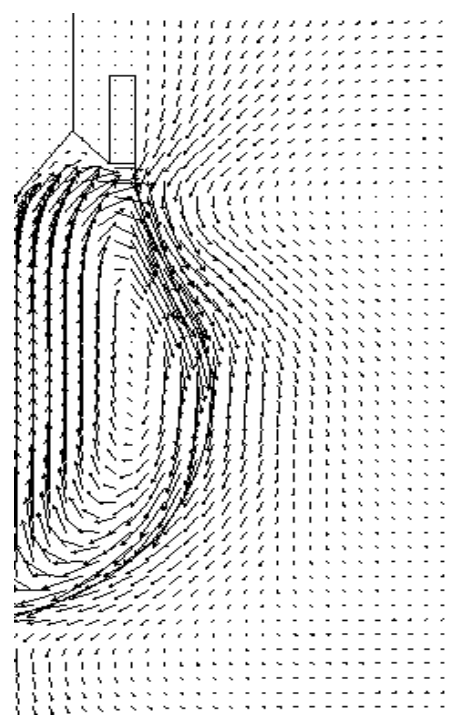

(b)



Fig. 6 : (a) Champ de vitesse total dans la goutte et dans la vapeur $\left(V_{\max }=8.8 \mathrm{~mm} / \mathrm{s}\right)$

(b) Contribution au changement de phase au champ de vitesse dans la vapeur (valeur max $_{1}=1 \mathrm{~mm} / \mathrm{s}$ )

La figure 6(b) montre que les conditions de pression dans l'enceinte ont été choisies afin d'obtenir un équilibre entre vaporisation sur la partie haute de l'interface et condensation sur sa partie droite. La comparaison du champ de vitesse dans la goutte et son intensité $\left(\mathrm{V}_{\max }=8.8 \mathrm{~mm} / \mathrm{s}\right)$ avec le champ de vitesse présenté sur la figure 5 (gauche) où le changement de phase était négligé, montre que l'écoulement dans la goutte n'est pas influencé pas ce phénomène qui reste donc négligeable.

\section{CONCLUSION}

Les simulations présentées ici, sur la configuration de la goutte pendante confinée dans une enceinte de vapeur, montrent que la modélisation numérique permet de fournir une vision précise des champs hydrodynamique et thermique dans la micropompe Marangoni, tant sur leur représentation spatiale que sur leur intensité. La modélisation devient ici un véritable outil d'expérimentation in silico et s'avère complémentaire d'une expérimentation difficile à mettre en œuvre dans les petits systèmes.

\section{REFERENCES BIBLIOGRAPHIQUES}

[1] P. Pham, 'Modélisation d'un dispositif de diagnostic moléculaire ultrasensible : étapes de concentration de nanoparticules superparamagnétiques et hybridation d' oligonucléotides sur support fonctionnalisé par micropompe Marangoni', Thèse de Doctorat, Institut National Polytechnique de Grenoble, 2001.

[2] C. Georgescu, 'Evolution d'une bulle : formation à partir d'un orifice et éclatement à la traversée d'une surface libre', Thèse de Doctorat, Institut National Polytechnique de Grenoble, 1999.

[3] L. Morino, 'Helmholtz decomposition revisited : Vorticity generation and trailing edge condition, part 1 : Incompressible flows', Computational Mechanics, 1, 65-90, 1986.

[4] J. Happel, H. Brenner, 'Low Reynolds number hydrodynamics', Martinus Nijhoff publishers, ISBN 90-247-2877-0, 1983.

[5] P. Massé, 'Analyse méthodologique de la modélisation numérique des équations de la physique des milieux continus à l'aide de la méthode des Eléments Finis. Flux-Expert : un système d'aide à la construction de logiciels' Thèse d'Etat, Institut National Polytechnique de Grenoble, 1983. 